\title{
Anthology of dermatopathological signs
}

\section{Anup Kumar Tiwary', Piyush Kumar²}

${ }^{1}$ Department of Dermatology, Subharti Medical College, Meerut, Uttar Pradesh, India, ${ }^{2}$ Department of Dermatology, Katihar Medical College, Katihar, India

Corresponding author: Dr. Anup Kumar Tiwary, E-mail: anup07tunnu07@gmail.com

\begin{abstract}
Dermatopathology is the keystone of dermatology that demands a thorough knowledge and a trained eye to accurately interpret the various microscopic changes and histopathological patterns. Therein, important diagnostics lie in guiding practicing dermatologists as well as postgraduate dermatology residents. This article makes assiduous effort to compile the important signs described by dermatopathology.
\end{abstract}

Key words: Sign; Mycosis fungoides; Eyeliner; Cookie cutter; Marquee

\section{INTRODUCTION}

Dermatopathology is the core subspecialty of dermatology, which has evolved intensively throughout the years. Numerous patterns and changes involve different layers of the skin, whose observation requires careful collection of biopsy samples, proper staining, and meticulous examination. There are many characteristic marks and signs reported by dermatopathology, which, if identified correctly, can be of remarkable importance for timely and precise diagnosis. Because no compilation of dermatopathological signs is to be found in databases such as PubMed, PubMed Central, SCOPUS, MEDLINE, EMBASE, and Web of Science, the following is an alphabetically-arranged compilation of the important signs described by dermatopathology.

1. Bare underbelly sign: In mycosis fungoides (MF), superficial perivascular lymphocytic infiltrates are concentrated more on the epidermal side of the superficial vascular plexus and the postcapillary venules than on the dermal side (Fig. 1). This sign appears inconsistently, but indicates lymphocytic epidermotropism, a major pathogenic process in mycosis fungoides [1].

2. Brass knuckles sign: Histopathologic examination of lobomycosis shows chains of thickly walled, yeastlike cells referred to as the brass knuckles sign [2].

3. Checkerboard sign: Histopathology of pityriasis rubra pilaris (PRP) shows alternations of orthokeratosis and parakeratosis in a vertical and horizontal direction resembling the checkerboard and, therefrom, referred to as the checkerboard sign [3].

4. Cookie-cutter sign: Magnified scans of punch biopsy specimens of the fully developed lesions of morphea show distinct straight lateral edges called cookie-cutter signs or squared-biopsy signs (Fig. 2) [1].

5. Cornflake sign: A ruptured epidermoid cyst that reacts as a foreign body with neutrophils, macrophages, and multinucleated giant cells, sometimes with the inclusion of scales, is known as the cornflake sign (Fig. 3) [4].

6. Eyeliner sign: Bowen's disease, also referred to as an in situ squamous cell carcinoma, is characterized by full-thickness epidermal atypia and complete loss of polarity in keratinocytes. This, coupled with the fact that the basement membrane remains intact and basal keratinocytes are spared, produces a windblown appearance known as the eyeliner sign (Fig. 4) [4].

7. Flag sign (also known as the pink-and-blue sign): This type of sign appears in actinic keratosis as alternating orthokeratosis above the spared ostia of acrosyringia and acrotrichia and parakeratosis above the interadnexal epidermis [5].

8. Floating sign: Histiocytes wrapping the collagen bundles of the middle and deep layers of the dermis, observed in morphea and interstitial granulomatous 
www.odermatol.com

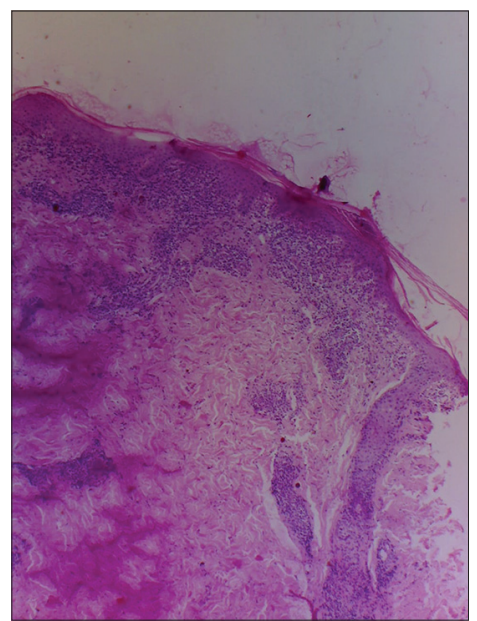

Figure 1: Localization of lymphocytes above the superficial vascular plexus (bare underbelly sign) (H\&E, $\times 100)$.

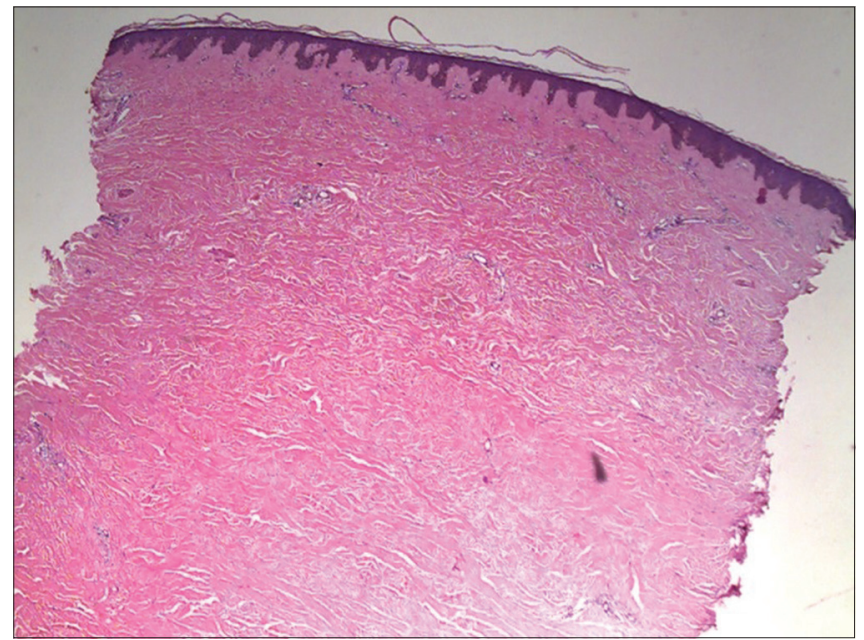

Figure 2: The cookie-cutter sign with sharp and straight edges (H\&E, $\times 100)$.

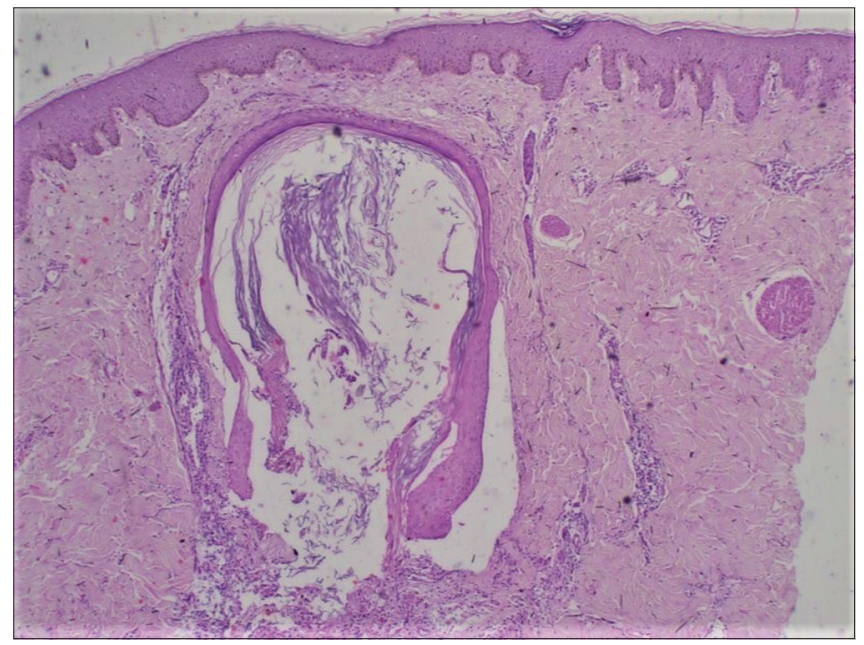

Figure 3: An epidermoid cyst $(H \& E, \times 200)$.

dermatitis, are known as the floating sign (Figs. 5 and 6) [6].

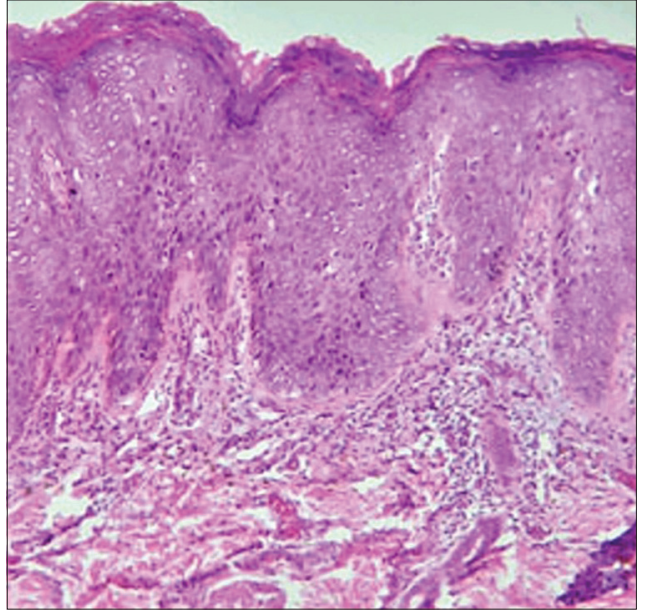

Figure 4: An intact basal layer and keratinocytes in Bowen's disease (H\&E, $\times 200)$.

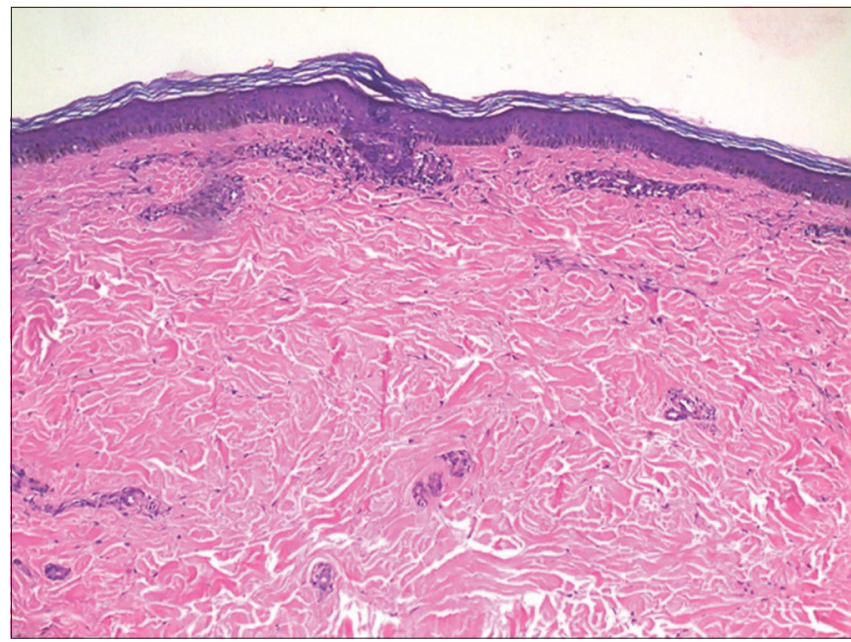

Figure 5: Histiocytes around collagen fibers in morphea (floating sign) (H\&E, ×200).

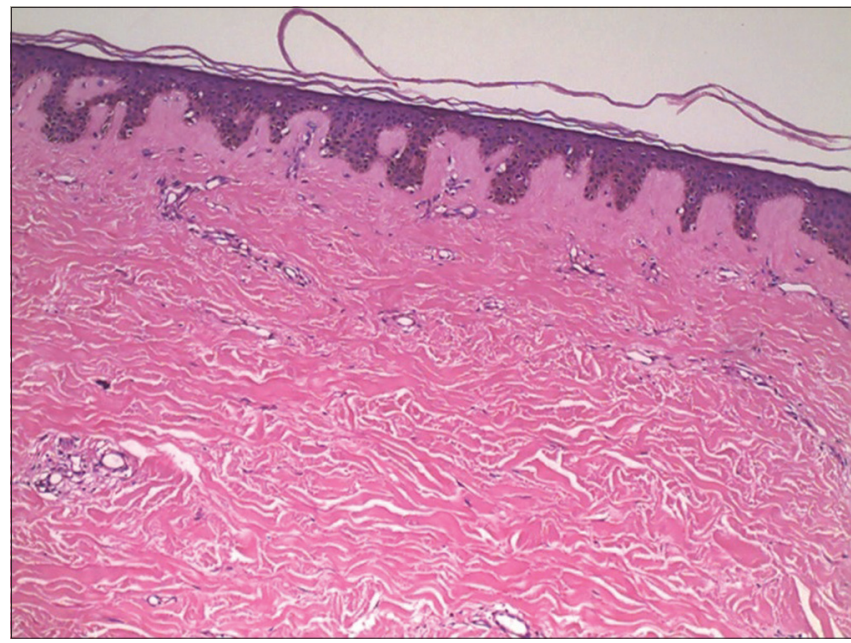

Figure 6: Another case of morphea with the floating sign $(H \& E, \times 200)$

9. Goggle sign: Central centrifugal cicatricial alopecia (CCCA), an example of lymphocytic 
scarring alopecia, involves perifollicular fibrosis of compound follicles, resembling the frame of a pair of glasses, and fused outer root sheaths, resembling the lenses. Other histological findings are follicular miniaturization, eccentric thinning of the follicular epithelium, premature desquamation of inner root sheaths, and loss of sebaceous glands [7].

10. Hairy palm sign: Histopathology of prurigo nodularis (PN) demonstrates thick compact orthohyperkeratosis, mimicking palmar skin histology (Fig. 7). The presence of folliculosebaceous units in prurigo nodularis reassures the diagnosis [8].

11. Hand-holding sign: A thickened interdigitating epidermis from the slightly tangentially cut biopsy sections of psoriasis forms the hand-holding sign (Fig. 8) [9].

12. Line sign: In morphea, the demarcation between the dermis and the subcutaneous layer becomes sharp and straight due to the loss of the rounded contour of subcutaneous fat lobules at the dermal interface and very dense collagen fibers [10].

13. Lowenbach's sign: The absence or attenuation of the granular cell layer in pityriasis rosea, first reported by Lowenbach, is known as Lowenbach's sign [11].

14. Marquee sign: In acute cutaneous leishmaniasis, amastigote leishmania can easily be seen at the periphery of macrophagic cytoplasm (Fig. 9). The marquee sign was named for its resemblance to the light bulbs arranged around a dressing room mirror [12].

15. Mesa sign: Papillomatous tips in epidermal nevi are flat-topped unlike in other papillomatous skin diseases with pointed or rounded tips, such as verruca vulgaris and seborrheic keratosis (Fig. 10) [1].

16. Murky sign: Merkel cell carcinoma is a deeply basophilic, highly cellular skin tumor with neuroendocrine differentiation. Histopathology reveals small- to medium-sized tumor cells with sparse cytoplasm and blurred cellular outlines, which is referred to as the murky sign [4].

17. Pizza sign: A trichoblastoma consists of two zones and types of neoplastic epithelial cells: the peripheral zone with dark palisading basophilic basaloid cells and the central zone with pale eosinophilic squamoid cells, a configuration known as the pizza sign [13].

18. Promontory sign: The patch and plaque stage of Kaposi's sarcoma (KS) has characteristic histopathological findings consisting of a dermal proliferation of irregular, jagged, thin-walled, dilated vessels lined with thin endothelial cells that surround

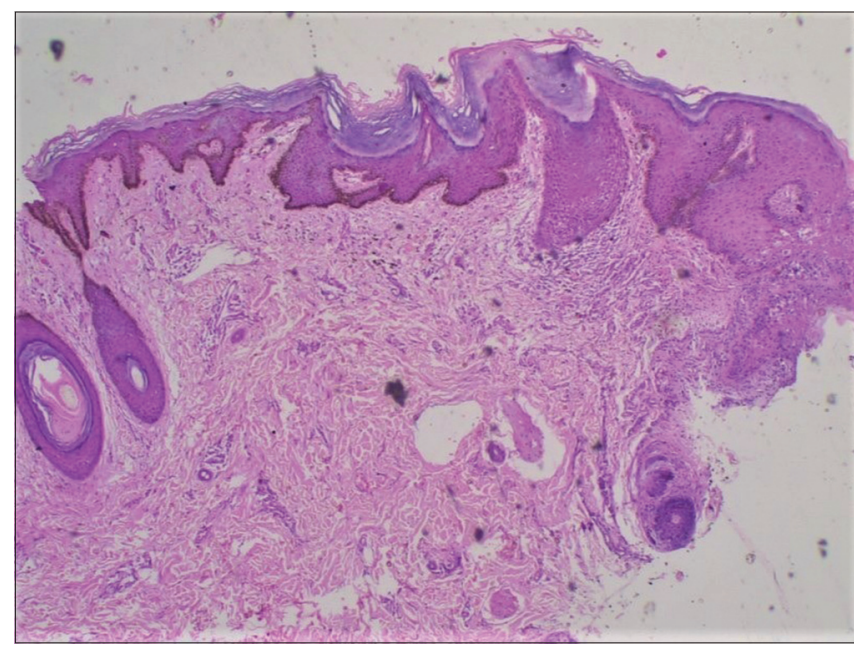

Figure 7: The hairy palm sign in thick compact orthohyperkeratosis (H\&E, $\times 200)$.

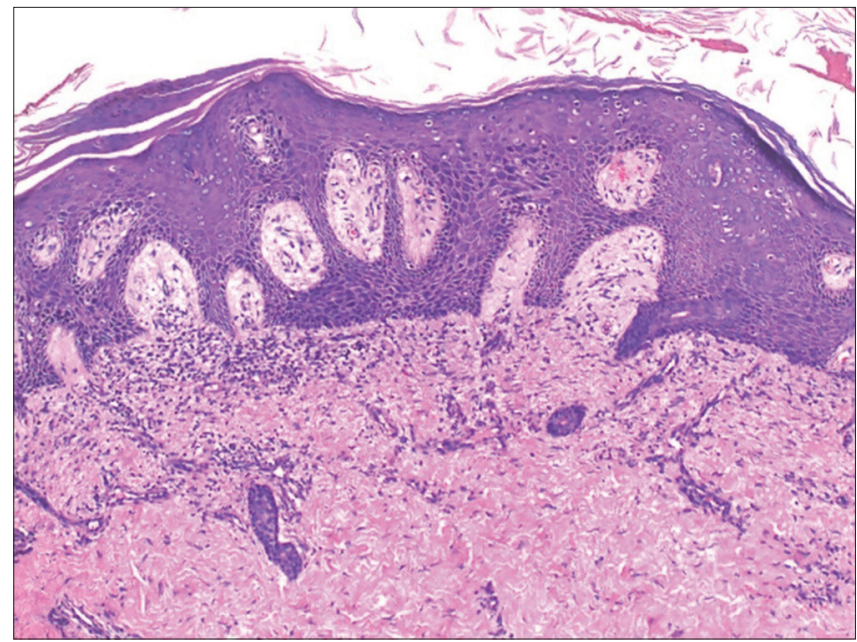

Figure 8: Histopathology of psoriasis showing an interdigitating acanthotic epidermis $(H \& E, \times 200)$.

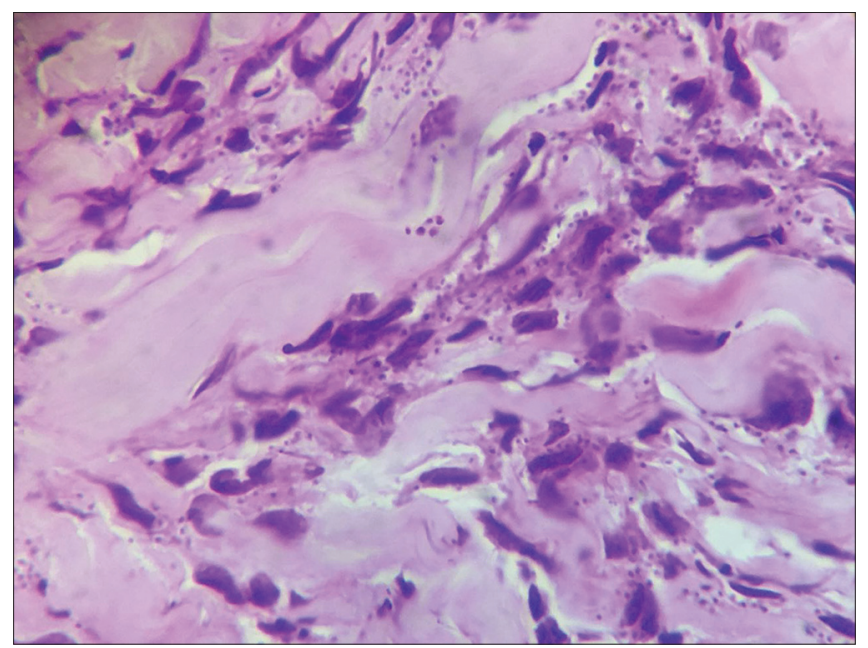

Figure 9: The marquee sign in cutaneous leishmaniasis (H\&E, $\times 400)$.

preexisting small vascular channels and spindle cells. The protrusion of the preexisting vessels into 
the surrounding dilated vascular space is referred to as the promontory sign, noted also in other vascular neoplasms, such as lymphangioendothelioma, retiform hemangioendothelioma, angiosarcoma, and spindle cell hemangioma [14].

19. Purple fiber sign: Elastotic fibers, which are usually admixed with the dermal component of melanocytes in acquired melanocytic nevi, often have a purple tincture. Absent in melanoma, this observation may help in differentiating benign and malignant melanocytic proliferations [15].

20. Recovery sign (also known as the last week's sign): In cured or treated cases of scabies, a normal lamellar stratum corneum with a basketweave pattern is seen underlying parakeratosis or orthokeratosis with mild dermal inflammation indicating prior inflammation [16].

21. Sabouraud's sign: This sign is an extravasation of erythrocytes in pityriasis rosea primarily into the papillary dermis and partly into the epidermis [11].

22. Salute sign (also known as the teapot spout sign or the teapot lid sign or the teapot sign): Pityriasis rosea involves an angulated parakeratosis attached at one end and free on the other, which very much resembles a salute, a sign that can also be seen in psoriasis, subacute eczema, and pityriasis lichenoides chronica (PLC) (Fig. 11) [11].

23. Sampaio sign: This sign is a manifestation of Pseudopelade of Brocq as a gelatinous mass observed close to the bulbar portion created by the removal of an atrophic plaque [17].

24. Sandwich sign: In dermatophytosis, fungi are seen in the stratum corneum, sandwiched between the upper zone of the normal basketweave layer and the lower zone of parakeratosis and orthokeratosis, sharing features of psoriasis such as psoriasiform epidermal hyperplasia and neutrophilic collections (Fig. 12) [14].

25. Screeching halt sign: Incompletely removed acquired melanocytic nevi (pseudomelanoma) reveals a melanocytic proliferation over the scarring. Interestingly, melanocytic nests do not spread beyond either end of the scarring, a pattern known as the screeching halt sign, also called the vertical line sign because both melanocytes and the scarring meet in the same place [14].

26. Signet-ring sign: Signet-ring cell carcinoma (SRCC) is an uncommon histological subtype of malignancy of the stomach, intestines, breasts, prostate, etc. Rarely, SRCC may metastasize to the skin and appear as nodules, giving a poor prognosis and being difficult to diagnose. Histopathology of nodular lesions shows that signet-ring cells appear

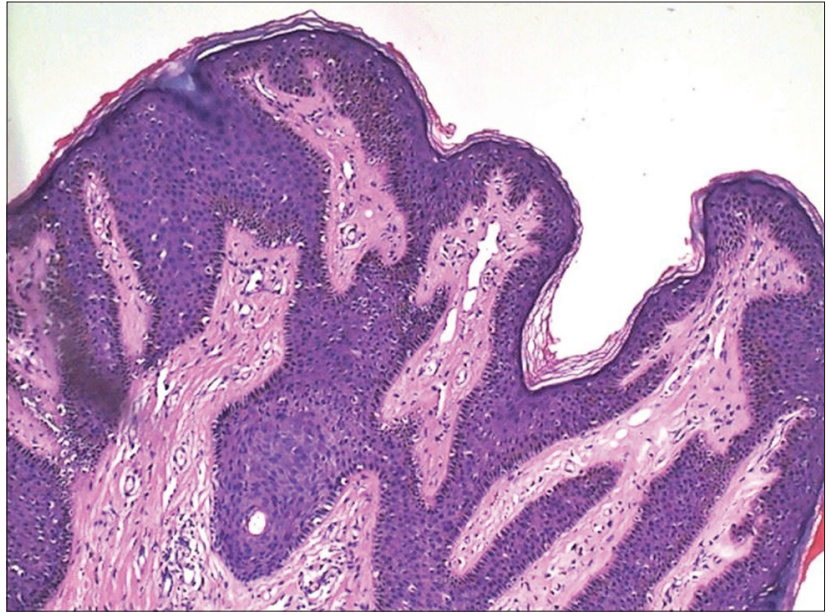

Figure 10: The flat-topped tips of the papillomatous epidermis of an epidermal nevus showing the mesa sign (H\&E, $\times 200)$.

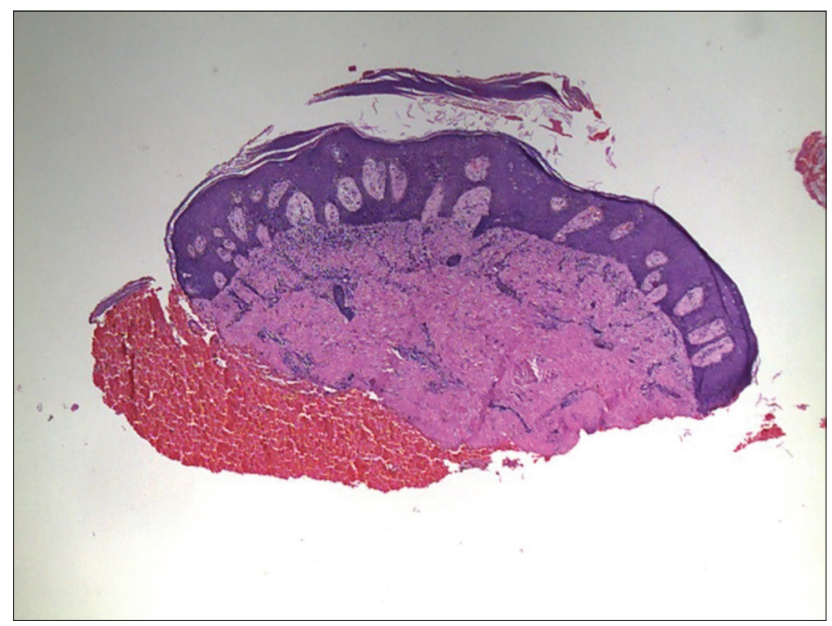

Figure 11: The salute sign in psoriasis $(H \& E, \times 100)$.

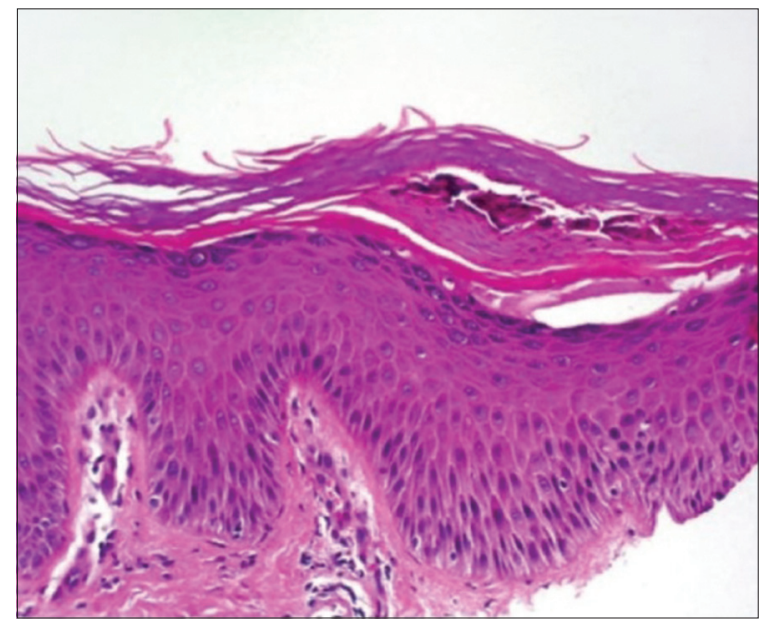

Figure 12: Fungal hyphae sandwiched between the upper normal and lower parakeratotic layers in dermatophytosis (H\&E, $\times 200)$.

because of the peripheral location of the nucleus from the accumulation of cytoplasmic mucins. This observation is referred to as the signet-ring sign, also 
observed in other conditions, such as erysipelas and contact dermatitis, and after formalin fixation as a cytoplasmic shrinkage artifact [18].

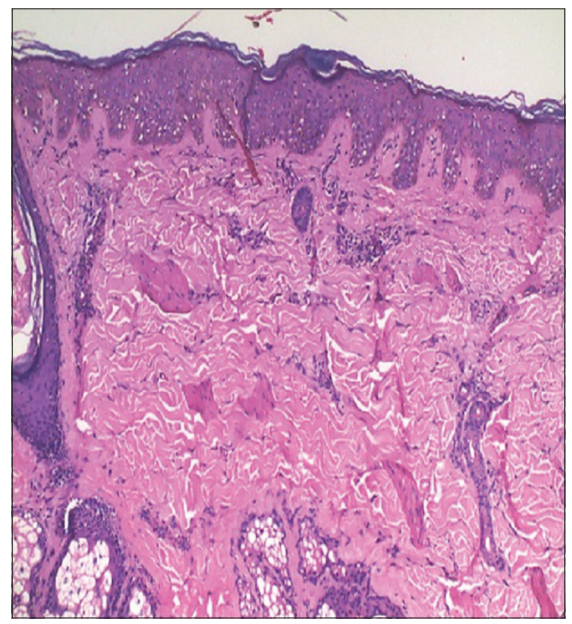

Figure 13: The swarm-of-bees sign (peribulbar lymphocytic infiltrates) in alopecia areata $(H \& E, \times 200)$.

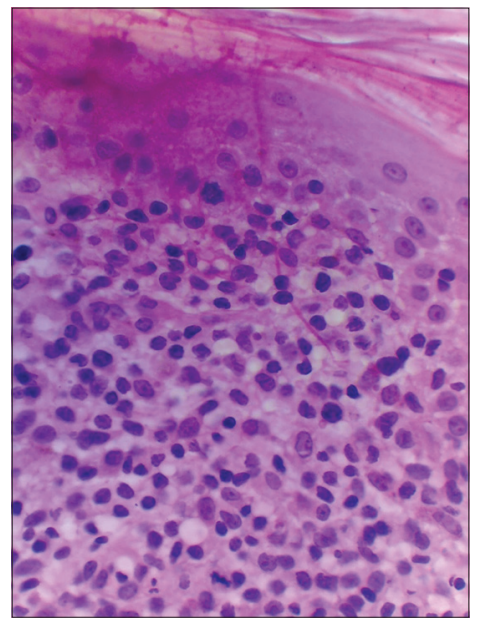

Figure 14: The toy soldier sign in neoplastic lymphocytes lined up along the DEJ in MF (H\&E, ×400).

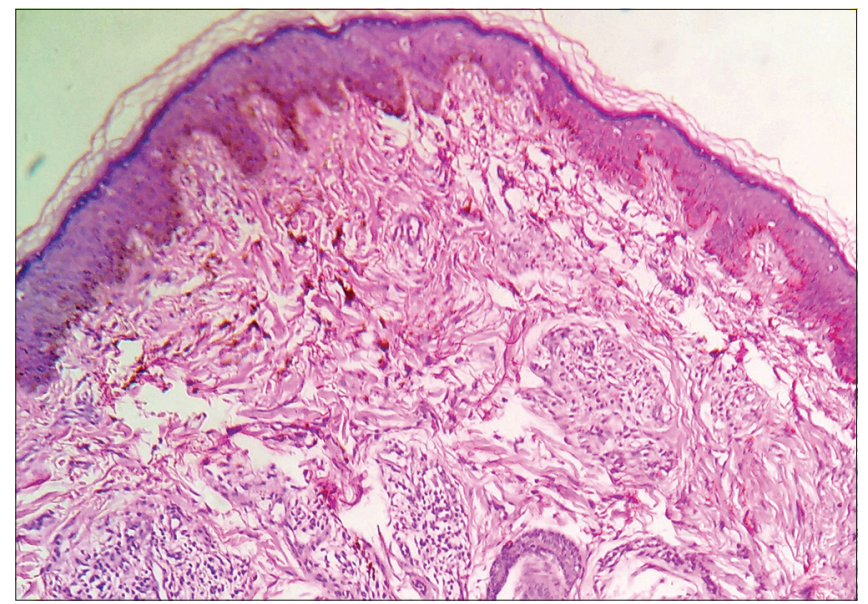

Figure 15: The umbrella sign in elastotic fibers below nevomelanocytes (H\&E, $\times 200)$.
27. Swarm-of-bees sign: Histopathologic examination of an active and expanding edge of a patch of alopecia areata shows follicles mostly in the late catagen or telogen phase, few in the anagen phase, situated in the subcutis or the middle layer of the dermis. The bulbs of these anagen follicles are surrounded by lymphocytic infiltrates and macrophages creating a pattern known as the swarm-of-bees sign (Fig. 13). The swarm-of-bees sign is usually observed in acute/ active inflammation in the anagen phase [17].

28. Toy soldier sign: Malignant Tlymphocytes in mycosis fungoides (MF) line up along the dermal-epidermal junction (DEJ) to produce a pattern known as the toy soldier sign (Fig. 14). These atypical, irregular lymphocytes may also invade the epidermis [19].

29. Tram track sign: In nephrogenic fibrosing dermopathy (NFD), also known as nephrogenic systemic fibrosis (NSF), plump CD34+ spindle or epithelioid cells with dendrites orient themselves parallel to elastic fibers and collagen bundles to produce a pattern known as the tram track sign [1].

30. Tricolor sign: This sign occurs in orf and Milker's nodules and owes its name to the three components in its structure: the red cornified layer, the white necrotic epidermis, and the blue basophilic dermal infiltrate.

31. Umbrella sign: Solar elastosis and melanocytic proliferations are common histological features of benign acquired melanocytic nevi and melanoma. The pattern of solar elastosis carries some diagnostic importance. In melanocytic nevi, elastic fibers are present in between melanocytes to receive protection from solar damage and are, for this reason, referred to as the umbrella sign (Fig. 15). This pattern is absent in melanoma, wherein elastotic fibers are pushed downward in the dermis by the expansion of malignant melanocytes [15].

32. Unna's sign: The eczematoid pattern in pityriasis rosea was first described by Unna in 1894 and is eponymously known as Unna's sign [11].

\section{Consent}

The examination of the patient was conducted according to the principles of the Declaration of Helsinki.

\section{REFERENCES}

1. Patterson JW. Weedon's Skin Pathology. $5^{\text {th }}$ edition. China: Elsevier Health Sciences; 2019.

2. Long V. Clothing-related Eponyms and Signs. Indian J Dermatol 2016;61(2):234.

3. Hanfstingl K, Pekar-Lukacs A, Motz R, Guenova E, Hoetzenecker 
W. Successful treatment of pityriasis rubra pilaris with ixekizumab. Case Rep Dermatol. 2018;2:10:97-100.

4. Kempf W, Hantschke M, Kutzner H, Burgdorf WHC. editors. Dermatopathology. Heidelberg: Springer;2008.

5. Cockerell CJ. Pathology and pathobiology of the actinic (solar) keratosis. Br J Dermatol. 2003;149:34-6.

6. Perez-Chua TA, Kisel YG, Chang KH, Bhawan J. Morphea and its variants and the "floating sign"-an additional finding in morphea. Am J Dermatopathol. 2014;36:500-5.

7. Miteva M. Non-inflammatory dermatoses. In: Miteva M. editor. A Notebook of Dermatopathology: Mastering the Basics, Pattern Recognition, and Key Pathologic Findings. $1^{\text {st }}$ ed. London: CRC Press; 2016.

8. Weigelt N, Metze D, Ständer S. Prurigo nodularis: Systematic analysis of 58 histological criteria in 136 patients. J Cutan Pathol. 2010;37:578-86.

9. Biswas A. Pearls and Pitfalls in Inflammatory Dermatopathology. $2^{\text {nd }}$ ed. Edinburgh: Cambridge University Press; 2017.

10. Fung MA. Inflammatory Diseases of the Dermis and Epidermis. In: Busam KJ. editor. Dermatopathology. $1^{\text {st }}$ ed. New York: Elsevier Health Sciences; 2010. p.74.

11. Thomas M, Khopkar U. Salute sign: A nonambiguous histopathological sign in pityriasis rosea. Indian Dermatol Online J. 2016;7:543-4.

12. Croitoru AGP, Chen HM, Ramos-e-silva M, Busam KJ. Infectious Diseases of the Skin. In: Busam KJ. editor. Dermatopathology. $1^{\text {st }}$ ed. New York: Elsevier Health Sciences; 2010. p.176.

13. Burg G, Kutzner H, Kempf W, Feit J, Smoller BR. Atlas of
Dermatopathology: Tumors, Nevi, and Cysts. $1^{\text {st }}$ ed. Hoboken, NJ: John Wiley \& Sons; 2018.

14. Madke B, Nayak C. Eponymous signs in dermatology. Indian Dermatol Online J. 2012;3:159-65.

15. Wood BA, Harvey NT. The "umbrella sign": a useful clue in the diagnosis of melanocytic lesions in sun damaged skin. Am J Dermatopathol. 2016;38:504-9.

16. Andrew Miner, Mariya Miteva. Key pathologic findings for levels of the skin. In: Mariya Miteva. editor. A Notebook of Dermatopathology: Mastering the Basics, Pattern Recognition, and Key Pathologic Findings. ${ }^{\text {st }}$ ed. London. CRC Press. 2016.

17. Rivitti Evandro A. Alopecia areata: a revision and update. An Bras Dermatol. 2005;80:57-68.

18. Gündüz Ö, Emeksiz MC, Atasoy P, Kidir M, Yalçin S, Demirkan S. Signet-ring cells in the skin: a case of late-onset cutaneous metastasis of gastric carcinoma and a brief review of histological approach. Dermatol Reports. 2017;4;8:6819.

19. Khopkar U, Doshi BR, Dongre AM, Gujral S. A study of clinicopathologic profile of 15 cases of hypopigmented mycosis fungoides. Indian J Dermatol Venereol Leprol. 2011;77:167-73.

Copyright by Anup Kumar Tiwary, et al. This is an open access article distributed under the terms of the Creative Commons Attribution License, which permits unrestricted use, distribution, and reproduction in any medium, provided the original author and source are credited.

Source of Support: Nil, Conflict of Interest: None declared. 